NASA/TM-1998-208961

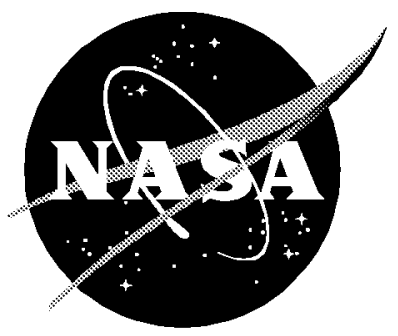

\title{
Tetrahedral Finite-Volume Solutions to the Navier-Stokes Equations on Complex Configurations
}

Neal T. Frink and Shahyar Z. Pirzadeh

Langley Research Center, Hampton, Virginia 


\section{The NASA STI Program Office ... in Profile}

Since its founding, NASA has been dedicated to the advancement of aeronautics and space science. The NASA Scientific and Technical Information (STI) Program Office plays a key part in helping NASA maintain this important role.

The NASA STI Program Office is operated by Langley Research Center, the lead center for NASA's scientific and technical information. The NASA STI Program Office provides access to the NASA STI Database, the largest collection of aeronautical and space science STI in the world. The Program Office is also NASA's institutional mechanism for disseminating the results of its research and development activities. These results are published by NASA in the NASA STI Report Series, which includes the following report types:

- TECHNICAL PUBLICATION. Reports of completed research or a major significant phase of research that present the results of NASA programs and include extensive data or theoretical analysis. Includes compilations of significant scientific and technical data and information deemed to be of continuing reference value. NASA counterpart or peer-reviewed formal professional papers, but having less stringent limitations on manuscript length and extent of graphic presentations.

- TECHNICAL MEMORANDUM. Scientific and technical findings that are preliminary or of specialized interest, e.g., quick release reports, working papers, and bibliographies that contain minimal annotation. Does not contain extensive analysis.

- CONTRACTOR REPORT. Scientific and technical findings by NASA-sponsored contractors and grantees.
- CONFERENCE PUBLICATION. Collected papers from scientific and technical conferences, symposia, seminars, or other meetings sponsored or co-sponsored by NASA.

- SPECIAL PUBLICATION. Scientific, technical, or historical information from NASA programs, projects, and missions, often concerned with subjects having substantial public interest.

- TECHNICAL TRANSLATION. Englishlanguage translations of foreign scientific and technical material pertinent to NASA's mission.

Specialized services that complement the STI Program Office's diverse offerings include creating custom thesauri, building customized databases, organizing and publishing research results ... even providing videos.

For more information about the NASA STI Program Office, see the following:

- Access the NASA STI Program Home Page at http://www.sti.nasa.gov

- Email your question via the Internet to help@sti.nasa.gov

- Fax your question to the NASA STI Help Desk at (301) 621-0134

- Telephone the NASA STI Help Desk at (301) 621-0390

- Write to: NASA STI Help Desk NASA Center for AeroSpace Information 7121 Standard Drive Hanover, MD 21076-1320 
NASA/TM-1998-208961

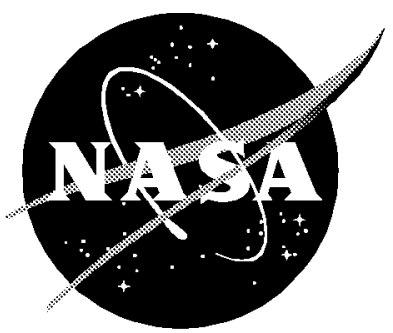

\section{Tetrahedral Finite-Volume Solutions to the Navier-Stokes Equations on Complex Configurations}

Neal T. Frink and Shahyar Z. Pirzadeh

Langley Research Center, Hampton, Virginia 
Available from:

NASA Center for AeroSpace Information (CASI) 7121 Standard Drive

Hanover, MD 21076-1320

(301) 621-0390
National Technical Information Service (NTIS) 5285 Port Royal Road

Springfield, VA 22161-2171 (703) 605-6000 


\section{ABSTRACT}

A review of the algorithmic features and capabilities of the unstructured-grid flow solver USM3Dns is presented. This code, along with the tetrahedral grid generator, VGRIDns, is being extensively used throughout the U.S. for solving the Euler and Navier-Stokes equations on complex aerodynamic problems. Spatial discretization is accomplished by a tetrahedral cell-centered finite-volume formulation using Roe's upwind flux difference splitting. The fluxes are limited by either a Superbee or MinMod limiter. Solution reconstruction within the tetrahedral cells is accomplished with a simple, but novel, multidimensional analytical formula. Time is advanced by an implicit backward-Euler time-stepping scheme. Flow turbulence effects are modeled by the Spalart-Allmaras oneequation model, which is coupled with a wall function to reduce the number of cells in the near-wall region of the boundary layer. The issues of accuracy and robustness of USM3Dns Navier-Stokes capabilities are addressed for a flat-plate boundary layer, and a full F-16 aircraft with external stores at transonic speed.

\section{INTRODUCTION}

This paper is offered in response to an invitation to present to the "finite element" community ${ }^{1}$ an alternate approach for solving the Navier-Stokes equations using a tetrahedral-based "finite volume" formulation. The focus will be on the features and application of the cell-centered upwind flow solver USM3Dns $[5,6,8]$ which is a component of the NASA Tetrahedral $U$ nstructured Software System $\left(\right.$ TetrUSS $\left.{ }^{2}\right)$ [7].

The primary attractiveness of tetrahedral-based schemes hinges on a demonstrated capability for rapid grid generation on a wide variety of complex geometries by a broad range of users [9,15,21]. The VGRIDns code [21], another component of TetrUSS, is widely used in the U.S. to generate inviscid and viscous tetrahedral grids on geometries ranging from aircraft to heart pumps. As with inviscid tetrahedral grids in the past, viscous tetrahedral grids are generated on complex geometries by a range of users in a matter of days.

The finite-volume approach is based on the physical concept of using macroscopic control volumes to numerically solve the conservation laws of fluid motion. Jameson, et. al. [12] reported one of the earliest successful implementations of this approach for solving the Euler equations on tetrahedral grids. The finite-element method [e.g. 18] is more mathematically based and uses a weak variational form of the governing equations, along with polynomial shape functions, for discretization. While there are inherent differences in the two methodologies, there are similarities between the Galerkin finite element procedure with piecewise-linear shape functions and the finite-volume approach [12].

Tetrahedral finite-volume Navier-Stokes methodology is maturing along two tracks: node-centered and cell-centered schemes, each with their relative merits. Node-centered schemes exploit an efficient edge-based data structure and more readily facilitate general polyhedral cell volumes [e.g. 2,4,16], but typically require large tetrahedral grids. Cell-centered schemes exploit geometric features of tetrahedra for constructing accurate spatial reconstruction schemes and provide comparable accuracy with fewer tetrahedra ${ }^{3}$, but they are not as easily extendable to general elements. While the underlying Navier-Stokes methodologies have advanced rapidly in recent years, they are still less mature than their more established "structured" grid counterparts. Legitimate questions still remain regarding the solution accuracy of unstructured Navier-Stokes schemes [3,4], and the user community does not yet have a sufficient experience base from which to derive full confidence. Thus, there is a strong need for more fundamental analyses and systematic application studies which address the key issues of solution accuracy, robustness, and efficiency on a range of configuration and flow classes.

There is an ongoing discussion among the computational fluid dynamics community regarding the use of pure tetrahedra vs. mixed elements for Navier-Stokes computations. While there has been no definitive resolution to this issue, the authors are generally pleased with the (cell-centered) tetrahedral approach. Grid generation is rapid and robust with VGRIDns. Grid sizes are manageable, and the number of tetrahedra needed to resolve the boundary layer is comparable to that for structured or prismatic grid methods. The primary shortcomings arise from the large memory requirements of general-indexed schemes using implicit time integration. Work is currently underway to resolve these problems using zonal decomposition techniques and adaptive gridding.

The scope of this paper is to review the underlying Navier-Stokes methodology of the VGRIDns grid generator and USM3Dns finite-volume flow solver, and to demonstrate this emerging capability on a very complex configuration. An assessment of solution accuracy is presented for the flat-plate boundary layer problem. The is-

\footnotetext{
${ }^{1}$ Tenth International Conference on Finite Elements in Fluids, Tucson, Arizona, USA, January 5-8, 1998

${ }^{2}$ URL: http://ad-www.larc.nasa.gov/tsab/tetruss

${ }^{3} \mathrm{~A}$ given tetrahedral grid has between $\mathbf{5}$ and $\mathbf{5 . 5}$ more tetrahedra than nodes. Additional spatial resolution is achieved by the cell- centered scheme on a given grid by virtue of resolving the flow solution at $>5$ times more spatial locations than node-centered scheme.
} 
sues of accuracy and robustness are examined with a transonic computation on a realistic complex configuration using a full F-16 aircraft with external stores.

\section{TETRAHEDRAL GRID GENERATOR, VGRIDns}

VGRIDns is based on the Advancing-Front (AFM) [14] and the Advancing-Layers (ALM) [20] methods. The generation of a 'Navier-Stokes' grid is accomplished automatically in three main steps: (1) generation of triangular surface grid by AFM and ALM, (2) generation of thin-layered tetrahedral grid in the boundary layer by ALM, and (3) generation of regular (inviscid) tetrahedral grid outside the boundary layer by AFM.

Grid clustering is controlled by a prescribed stretching function within the 'viscous' layers, and a 'transparent' Cartesian background grid [19] overlaying the entire domain. Included in the background grid are a number of prescribed 'point' and 'line' sources for defining local cell spacings. The grid characteristics are smoothly diffused from the sources onto the background grid nodes by solving an elliptic equation. The problem is analogous to the transfer heat in a conducting medium.

Two main operations are involved in ALM: 1) computation of surface vectors along which the grid points are distributed and 2) construction of a pattern for a compatible cell connectivity within the thin layers. Thin layers of tetrahedra are formed by inserting new points along the surface vectors and connecting the points according to the predetermined connectivity pattern. The individual layers (see Fig. 1) continue advancing independent of each

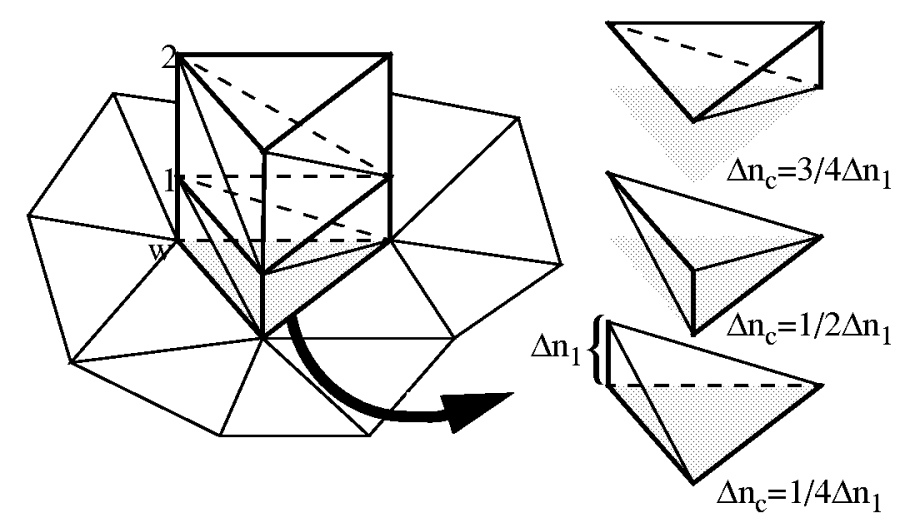

Fig. 1 Thin-layered tetrahedra formed by ALM; $\left(\Delta \mathbf{n}_{1}\right.$ - height of first node, $\Delta \mathbf{n}_{c}$ - height of cell centroid).

other until either the background grid information or an approaching front warrants them to stop. When all layers are complete, the ALM process automatically switches to the AFM to generate regular tetrahedra outside the boundary layer. With a common background grid controlling both methods, the transition from thin layers to the regular grid becomes gradual and continuous.

Another feature of VGRIDns which is of practical importance for Navier-Stokes is its ability to generate multi-directional anisotropically stretched grids [22]. This results in at least a factor of three reduction in overall number of cells. With such a capability, fewer points are distributed in the directions of reduced flow gradient with no loss of grid resolution in other essential direction(s).

\section{FINITE-VOLUME FLOW SOLVER, USM3Dns}

The fluid motion is governed by the time-dependent Reynolds-averaged Navier-Stokes equations for an ideal gas which express the conservation of mass, momentum, and energy for a compressible Newtonian fluid in the absence of external forces. The equations are prescribed in integral form for a bounded domain $\Omega$ with the boundary $\partial \Omega$

$$
\frac{\partial}{\partial t} \iiint_{\Omega} Q d V+\iint_{\partial \Omega} \boldsymbol{F}(Q) \cdot \hat{\boldsymbol{n}} d S=\iint_{\partial \Omega} \boldsymbol{G}(Q) \cdot \hat{\boldsymbol{n}} d S
$$

where the state variables are $Q=\left[\rho, \rho u, \rho v, \rho w, e_{o}\right]^{T}$, and the inviscid and viscous fluxes, $\mathbf{F}(\mathbf{Q})$ and $\mathbf{G}(\mathbf{Q})$, 
respectively, are defined in Ref. [8].

A finite-volume discretization is applied to Eq. 1 which results in a consistent approximation to the conservation laws. The spatial domain is divided into a finite number of tetrahedral cell volumes. The discretized solution to Eq. 1 results in a set of volume-averaged state variables which are in balance with the area-averaged fluxes (inviscid and viscous) across the cell faces.

\section{Inviscid Fluxes}

The primary challenge in designing a tetrahedral cell-centered finite-volume scheme is the accurate reconstruction of cell-averaged data within non-isotropic tetrahedra to the perimeter triangular faces for flux computation as illustrated in Fig. 2. A novel cell reconstruction process was derived in Ref. [6], which is based on an ana-

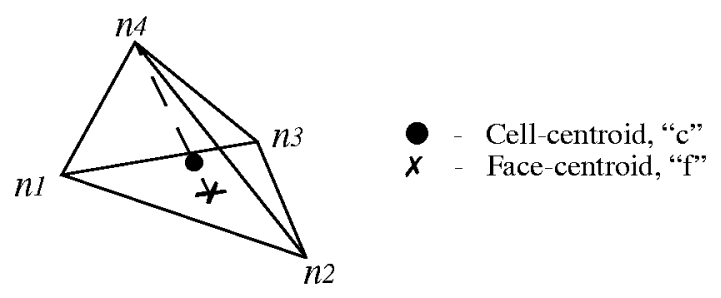

Fig. 2 Reconstruction stencil for tetrahedral cell-centered scheme.

lytical formulation for computing the gradient term of a Taylor series expansion within tetrahedral cells. The scheme consists of simple, universal formula for tetrahedral cells

$$
\boldsymbol{q}_{f(1,2,3)}=\boldsymbol{q}_{c}+\frac{1}{4}\left[\frac{1}{3}\left(\boldsymbol{q}_{n 1}+\boldsymbol{q}_{n 2}+\boldsymbol{q}_{n 3}\right)-\boldsymbol{q}_{n 4}\right]
$$

where $q=[\rho, u, v, w, p]^{\mathrm{T}}$ are the primitive variables of density, velocity, and pressure. Its derivation is based on exploiting several invariant features of tetrahedra in combination with the mid-point trapezoidal rule for numerical integration. This gives rise to the analytical expression of the Taylor series expansion of the cell-averaged solution to the cell face in Eq. 2. With an accurate reconstruction of data at the cell faces, the inviscid flux quantities are computed in a straightforward manner across each face using the Roe [23] flux-difference splitting (FDS) approach. Limiting of the reconstructed solution is generally necessary for FDS to eliminate overshoots of the expansion in high-gradient regions, such as shocks. The well known Superbee or MinMod limiters have been implemented in USM3Dns.

A key component of the scheme is the secondary reconstruction of surrounding cell-averaged data to a common vertex or node by a weighted averaging procedure. The current averaging scheme, originally proposed in Ref. [5], is based on an inverse-distance weighting of the primitive variables from the cell centroid to the cell vertices:

$$
\boldsymbol{q}_{n}=\left(\sum_{i=1}^{N} \frac{\boldsymbol{q}_{c, i}}{r_{i}}\right) /\left(\sum_{i=1}^{N} \frac{1}{r_{i}}\right)
$$

where $r_{i}=\left[\left(x_{c, i}-x_{n}\right)^{2}+\left(y_{c, i}-y_{n}\right)^{2}+\left(z_{c, i}-z_{n}\right)^{2}\right]^{1 / 2}$.

Reconstruction at boundary nodes is accomplished through ghost-cells as described in Ref. [6].

Until recently, the sole approach for secondary reconstruction was a pseudo-Laplacian averaging scheme presented in Ref. [6]. This scheme offers the advantage of second-order accuracy in reconstructing data from surrounding cells to a node. However, there is a need to artificially "clip" the weighting factors between 0 and 2 (Ref. [10]) to avert a violation of the positivity principle which is necessary for solution stability. This artificial "clipping" process does, unfortunately, compromise the formal second-order accuracy of the scheme to some extent. Recent experiences with applying the pseudo-Laplacian scheme to Navier-Stokes computations have surfaced some anomalous behavior which needs further investigation. Meanwhile, for the present work, we are temporarily 
reverting to the inverse-distance averaging of Eq. 3 which is less accurate, but will never violate the principle of positivity.

\section{Viscous Fluxes}

The viscous fluxes $G(Q)$ are approximated at the cell-face centroids by linear reconstruction which provides a continuous representation of the solution variables across the cell faces. A modified version of the stencil presented by Mitchell [17], sketched in Fig. 3, utilizes the averaged solution quantities at the three vertices of a cell face, $\mathbf{q}_{\mathbf{n} 1}, \mathbf{q}_{\mathbf{n} 2}$, and $\mathbf{q}_{\mathbf{n} 3}$, and the cell-centered values of the two cells sharing the face, $\mathbf{q}_{\mathbf{c 1}}$ and $\mathbf{q}_{\mathbf{c 2}}$, where $\mathrm{q} \equiv(\rho, u, v, w, p)$. The required derivatives $u_{x}, u_{y}$, etc. for $G(Q)$ are derived from a Cramer's rule solution to

$$
\left[\begin{array}{cc}
x_{c 2}-x_{c 1} & y_{c 2}-y_{c 1} \\
\left(0.5\left(x_{n 2}+x_{n 3}\right)-x_{n 1}\right) & \left(0.5\left(y_{n 2}+y_{n 3}\right)-y_{n 1}\right)\left(0.5\left(z_{n 2}+z_{n 3}\right)-z_{n 1}\right) \\
\left(0.5\left(x_{n 3}+x_{n 1}\right)-x_{n 2}\right) & \left(0.5\left(y_{n 3}+y_{n 1}\right)-y_{n 2}\right)\left(0.5\left(z_{n 3}+z_{n 1}\right)-z_{n 2}\right)
\end{array}\right]\left[\begin{array}{l}
u_{x} \\
u_{y} \\
u_{z}
\end{array}\right]=\left[\begin{array}{c}
u_{c 2}-u_{c 1} \\
\left(0.5\left(u_{n 2}+u_{n 3}\right)-u_{n 1}\right) \\
\left(0.5\left(u_{n 3}+u_{n 1}\right)-u_{n 2}\right)
\end{array}\right]
$$

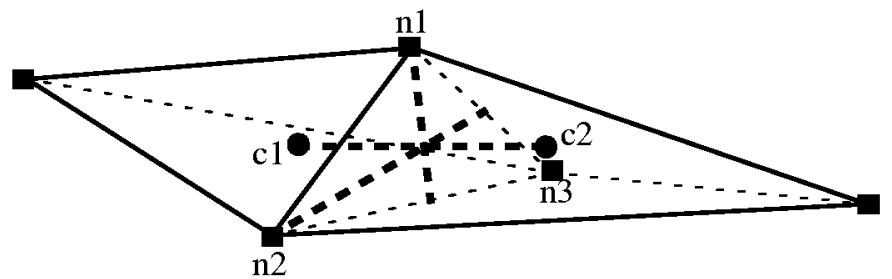

Fig. 3 Modified tetrahedral viscous stencil.

\section{Time Integration}

The computations are advanced to steady state by the implicit time advancement strategy of Anderson [1]. The scheme uses the linearized, backward Euler time differencing approach to update the solution at each time step. The linear system of equations are solved at each time step with a point-Jacobi subiteration on groups of tetrahedral cells which are separated into 'colors' (different from face-coloring) such that no two cells share a common face. While the point-Jacobi method is in itself not very efficient, convergence rate is accelerated by using the latest values of the updated solution variables as soon as they are available after subiteration of prior 'colors'. This produces a Gauss-Seidel-like effect, and the method has the advantage of being completely vectorizable. Typically, 10 subiterations are used for Navier-Stokes computations, and 20 subiterations for Euler.

Because of the number of operations required to invert a matrix depends on the matrix bandwidth, the lefthand side of the system of linear equations is evaluated with first-order differencing to reduce both required storage and computer time. Convergence of the subiterations is further accelerated by using Van Leer's Flux Vector Splitting (FVS) [25] on the left-hand side. Thus in the present study, first-order differencing and FVS are applied to the left-hand side, and higher order differencing and FDS to the right-hand side. The viscous Jacobian terms are included in the left-hand side of the equation.

USM3Dns requires 175 words/cell of core memory, and runs at a speed of $27 \mu \mathrm{s} /$ cell/cycle on a CRAY C90 with higher-order differencing. Multitasking on the CRAY is utilized for reducing the wall-clock time of a computation. Multitask efficiencies of 6 out of 10 processors on a 16-processor machine are typically achieved for most applications.

\section{Turbulence Model}

Spalart-Allmaras. Closure of the Reynolds stress is provided by the one-equation Spalart-Allmaras (S-A) turbulence model. This model is derived "using empiricism and arguments of dimensional analysis, Galilean invariance, and selective dependence on the molecular viscosity" [24]. The model solves a partial differential equation (PDE) over the entire field for a transformed working variable $\tilde{v}$ from which the eddy viscosity, $\mu_{t}$, can be extracted. The PDE is solved separately from the flow equations using the same backward Euler time integration 
scheme, which results in a loosely coupled system. The production and destruction terms have been modified as recommended in Ref. [24] to ensure positive eddy viscosity throughout the computation.

On 'no-slip' surfaces, the dependent variable, $\tilde{v}$, is set to zero. For tangent-flow surfaces, a zero gradient of the variable is applied. Far field boundary conditions are applied by extrapolating $\tilde{v}$ from the interior for outflow boundaries, and taken from the free stream for the inflow.

The S-A model requires that the distance of each cell to the nearest wall be provided for the near-wall damping terms for cells which are in proximity to 'viscous' surfaces. These distances are determined prior to code execution for cells in the "viscous" layers. A very efficient preprocessing code is developed which exploits the ordering of the nodes in these layers (see Fig. 1) to complete the distance computation within a few seconds.

Wall Function. The S-A model has been coupled with a wall function formulation to eliminate the need for resolving the flow in the sublayer portion of a turbulent boundary layer. Details are presented in Ref. [8]. With this approach, the inner region of the boundary layer is modeled by an analytical function which is matched with the numerical solution in the outer region. This has the advantage of 1) significantly reducing memory requirement by eliminating a large portion of cells normally required to resolve the sublayer, and 2) improving overall convergence by removing the thinner, more highly stretched cells which add stiffness to the solution process.

The selected wall function is a law-of-the-wall expression [26] derived by Spalding in 1961. The present implementation considers no adjustment to adiabatic wall density, which is important to high speed flows.

\section{RESULTS AND DISCUSSION}

Results are presented in this section for the flat-plate boundary layer problem, and a complete F-16 aircraft with external stores at transonic speeds. Only a cursory summary of the flat-plate problem is included from prior work [8] to review the accuracy of a relatively new wall-function approach. The F-16 computation was chosen to demonstrate the emerging unstructured finite-volume Navier-Stokes capability on a realistic complex aircraft configuration which exhibits strong viscous effects during store separation.

\section{Grid Spacing}

The normal grid spacing across the boundary layer is prescribed by the exponential function

$$
\Delta n_{j}=\Delta n_{1}\left(1+a(1+b)^{j-1}\right)^{j-1}
$$

such that the distance from the $j^{\text {th }}$ layer to the surface is given by $n_{j}=n_{j-1}+\Delta n_{j-1}$.

The parameter $\Delta \mathrm{n}_{1}$ is the spacing of the first node above the surface (Fig. 1), while $a$ and $b$ are parameters which govern the growth. A small utility program is used to determine an initial estimate of $\Delta \mathbf{n}_{1}, a$, and $b$ on an assumed 1/7th law velocity profile for prescribed values of Reynolds number, $\mathrm{n}^{+}$, and number of points across the boundary layer.

\section{Flat-Plate Boundary Layer}

The flat-plate boundary layer solution is used to assess the accuracy of the wall function in predicting the flat-plate turbulent skin friction. The computations were made on quasi- $2 \mathrm{D}$ tetrahedral 'channel' grids for $\mathrm{M}_{\infty}=0.5$ and $\mathrm{Re}_{\mathrm{L}}=2 \times 10^{6}$ where " $\mathrm{L}$ " is the length of the plate.

Grid 1 was generated by constructing a 49X12 H-topology structured grid with a normal spacing defined by $\Delta \mathrm{n}_{1}=0.001 \mathrm{~L}, a=0.3$, and $b=0.07$ in Eq. 5 , which yields roughly 5 nodes across the boundary layer at $\mathrm{x} / \mathrm{L}=0.5$ and an approximate $\mathrm{n}^{+}$of 80 at the first node. The resulting upper domain boundary $(\mathrm{k}=12)$ is located at $0.22 \mathrm{~L}$. The $2 \mathrm{D}$ grid was stacked spanwise in $0.02 \mathrm{~L}$ increments to form three planes resulting in a 3D structured dualchannel grid (49X3X12) of H-H topology. Each hexahedral cell was subdivided into 2 prismatic cells, which were further subdivided into 3 tetrahedra each to form the 3D tetrahedral grid with 6,336 cells. The "flat plate" was discretized by a cosine clustering between the "structured" indices $15 \leq i \leq 49$ along the $k=1$ boundary with inviscid flow prescribed on the $k=1$ boundary ahead of the plate. Boundary conditions of constant entropy and constant total pressure were prescribed on the inflow plane, while an extrapolation condition was applied to the upper and exit domain boundaries. A constant freestream pressure was also imposed on the exit plane.

A second grid was generated in a similar manner as the first to explore the lower limits of grid coarseness on solution accuracy. Grid 2 was constructed from a $49 \mathrm{X} 6 \mathrm{H}$-topology with the Eq. 5 parameters of $\Delta \mathrm{n}_{1}=0.001 \mathrm{~L}$, 
$a=2.0$, and $b=0.07$. This resulted in a 3D channel grid (49X3X6) with 2,880 cells, and an upper domain boundary $(\mathrm{k}=6)$ also at $0.22 \mathrm{~L}$.

Fig. 4 portrays the effect of normal grid density on skin-friction coefficient and the law-of-the-wall behavior at $\mathrm{x} / \mathrm{L}=0.5, \operatorname{Re}_{\mathrm{x}}=1 \times 10^{6}$, for the two grids. The plotted nodal solutions were reconstructed from the surrounding
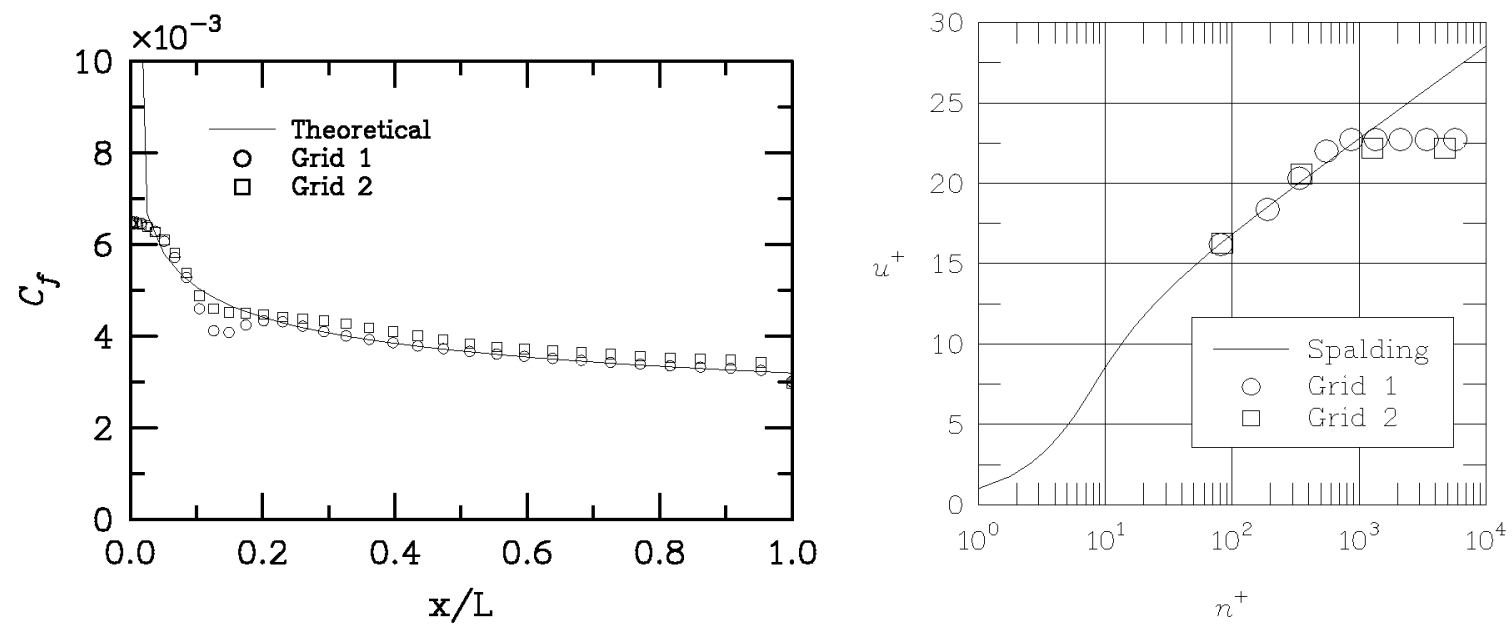

Fig. 4 Effect of normal grid density on skin friction (left), and law-of-the-wall at $\mathbf{x} / \mathrm{L}=\mathbf{0 . 5}$ (right), for flat-plate boundary layer flow; Mach $=0.5$ and $\operatorname{Re}_{\mathrm{L}}=2$ million.

tetrahedral cells using the weighted averaging procedure of Ref. [6]. The plotted nodal data points effectively have three layers of tetrahedra between each pair, as sketched in Fig. 1. Note that the first nodal value is matched with the $\log$ layer at approximately $n^{+}=80$ for both grids. Grid 1 has 5 nodes ( 15 tetrahedra) across the boundary layer, while Grid 2 has 3 nodes ( 9 tetrahedra). Grid 1 displays excellent agreement over $0.2<\mathrm{x} / \mathrm{L} \leq 1.0$ with the theoretical coefficient for fully turbulent flow, $C_{f}=0.0583\left(R e_{x}\right)^{-1 / 5}$, which is based on the $1 / 7$ th power law assumption. Grid 2 does not exhibit the same level of agreement, but is remarkably close considering its extreme grid coarseness across the boundary layer.

These results highlight a common misconception about tetrahedral grid resolution. For a cell-centered scheme, it is important to think in terms of cell-resolution rather than node-resolution. It is the number of cells across the boundary layer that are important to solution accuracy for the present method.

On a side note, the spurious behavior of the computed skin friction near the plate leading edge $(0 \leq x<0.2)$ in Fig. 4 is not fully understood at this time and is presently under investigation. However, the principal interest for the present study is in the fully developed turbulent flow over the remaining region of the plate.

\section{F-16 with Generic Finned Store}

Unstructured transonic Navier-Stokes computations are presented for the complete F-16 aircraft configuration as a demonstration and assessment of the present finite-volume methodology for a very complex geometry and flow field. In addition to the base aircraft, the configuration includes a flowing inlet, external fuel tank mounted on the inner wing pylon, and a generic finned-store suspended below the outer wing pylon. Support stings are also attached to the fuselage and the generic finned-store to simulate the wing-tunnel configuration tested in Ref. [11]. The horizontal and vertical tails were not present during the wind-tunnel test, but are included on the computational geometry as additional geometric complexities for demonstration purposes. Their influence on the store pressures is expected to be small.

An inviscid Euler study on this configuration is reported by Kern and Bruner [13] where the primary interest was the accurate and efficient prediction of initial loads on the finned-store to drive a separation trajectory analysis. The format of the following results is replicated from Ref. [13] to better complement the existing knowledge base. 
Grid Generation. Tetrahedral viscous and inviscid grids were generated for the F-16 aircraft using VGRIDns. The 'viscous' grid, shown in Fig. 5, has 1,428,779 tetrahedra, 255,959 nodes, whereas the 'inviscid' grid (not shown) contained 1,111,762 cells and 202,378 nodes. The farfield boundaries were prescribed as 5.4,6.2, and 3.6 body lengths ahead, aft, and spanwise of the aircraft, respectively. These boundary locations respectively correspond to 25, 29, and 17 wing mean-aerodynamic-chord (mac) lengths.
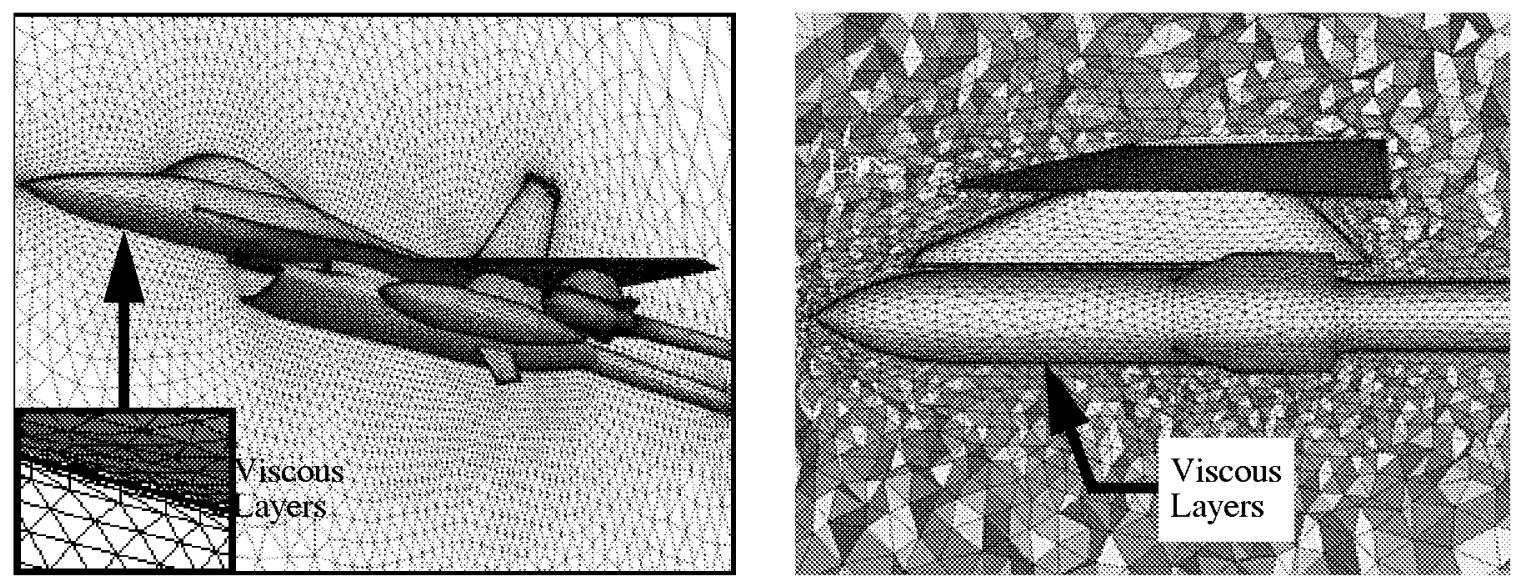

Fig. 5 Viscous tetrahedral grid on complete F-16 aircraft with external stores, 1,428,779 cells. (Left - triangulation of surface and symmetry plane, Right - tetrahedra on plane intersecting wing/pylon/finned-store.)

The F-16 surface definition is defined in full-scale inches. The normal grid spacing for the Navier-Stokes computation is sized for a wind-tunnel Reynolds number of 2 million, based on mac, assuming a 1/7th law boundary layer profile at the mid-mac location on the wing. The spacing is prescribed for computation with the wall function to yield 18 tetrahedral layers ( 6 nodes) across the mid-chord boundary layer with an $\mathrm{n}^{+}=30$ for the first node. The corresponding parameters for Eq. 5 are $\Delta \mathrm{n}_{1}=0.046$ inches, $a=0.745$, and $b=0.07$. The thin-layered tetrahedra are evident on the symmetry plane in Fig. 5 and on a plane cutting through the wing, pylon, and store. While the grid was generated over an extended period of time, it is estimated that the cumulative manual labor was in the range of 40 to 60 hours.

The 'inviscid' grid was generated from the same 'viscous' grid input file with an initial grid spacing of $\Delta \mathbf{n}_{1}=0.2$ which produced a near identical match between the two surface triangulations. Inviscid grids are typically generated entirely by the AFM approach. However, to gain more control of the cells generated within the small gap between the finned-store and its pylon, it was necessary to conduct the inviscid grid generation with the ALM option using an appropriate initial spacing which yielded five layers of tetrahedra within the gap.

Flow Solution. Navier-Stokes (N-S) and Euler flow solutions were obtained for angles of attack (AOA) of $0,2,4$, and 8 degrees at Mach 0.95. The Navier-Stokes cases were run for 1000 iterations starting from freestream conditions with an initial Courant-Friedrichs-Lewy (CFL) number of 0.01 that was ramped up to 100 over $500 \mathrm{cy}-$ cles. The Euler solutions were run for 800 cycles starting from freestream with an initial $\mathrm{CFL}=10$ with ramping up to 50 over 50 cycles. Convergence was accelerated to steady state with local time stepping. The inviscid fluxes were limited with the Superbee limiter.

The USM3Dns solver was executed with multitasking over 10 processors on a 16-processor CRAY C90. The Navier-Stokes and Euler solutions required 254 and 197 megawords of core memory, respectively. With a multitasking efficiency of 6 out of 10 processors, each N-S solution required from 10.5 to 11 hours of CPU time which was turned around in 2 wallclock hours. One N-S solution required $14.8 \mathrm{CPU}$ hours and 2.75 wallclock hours due to the particular scheduling load of the multitasking queue when the run was submitted. The Euler solutions each utilized approximately 6.6 CPU hours and 45 wallclock minutes of CRAY time, with a multitasking efficiency of 8.7 out of 10 processors.

The history of normalized residual error and lift coefficient for the Navier-Stokes solutions in Fig. 6 show a consistent convergence for each case. The leveling off of residual error is common for solutions on complex configurations at transonic speeds which also occurred for the Euler solutions. The oscillatory behavior above 500 iterations is most likely due to local recirculatory flow oscillations. Convergence is usually assessed from the force and moment coefficients. 

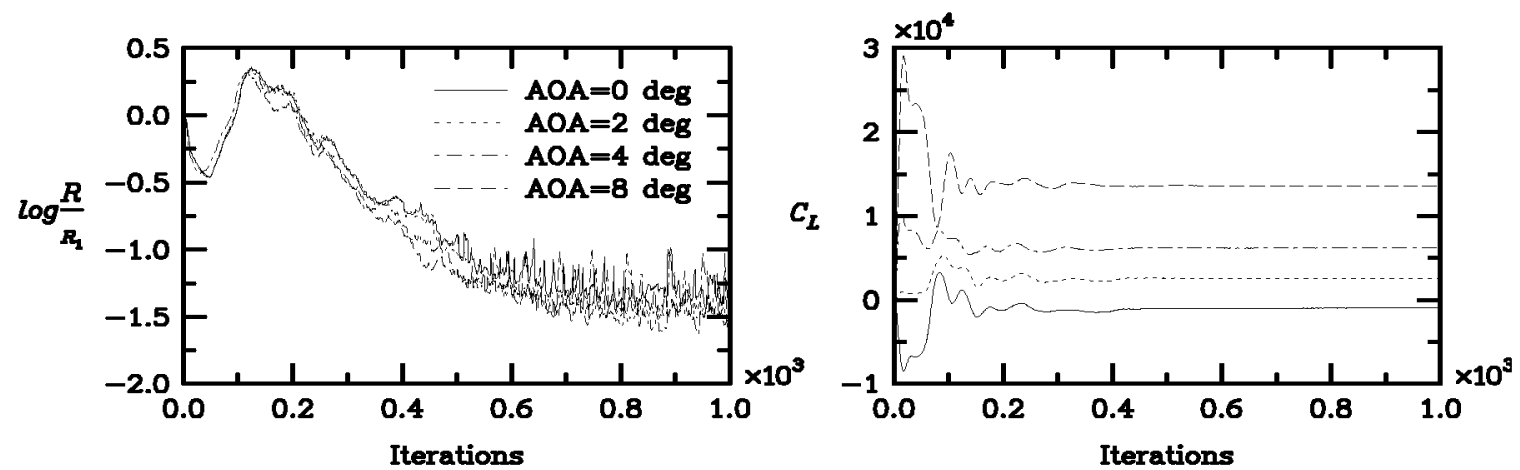

Fig. 6 History of L2-norm residual error and lift coefficient of Navier-Stokes flow solutions.

Figure 7 depicts the surface pressure contours and store/pylon flow patterns for the N-S solution at $\mathrm{AOA}=4$ degree. The global pressure field is characterized by strong shock systems and interactions, both between
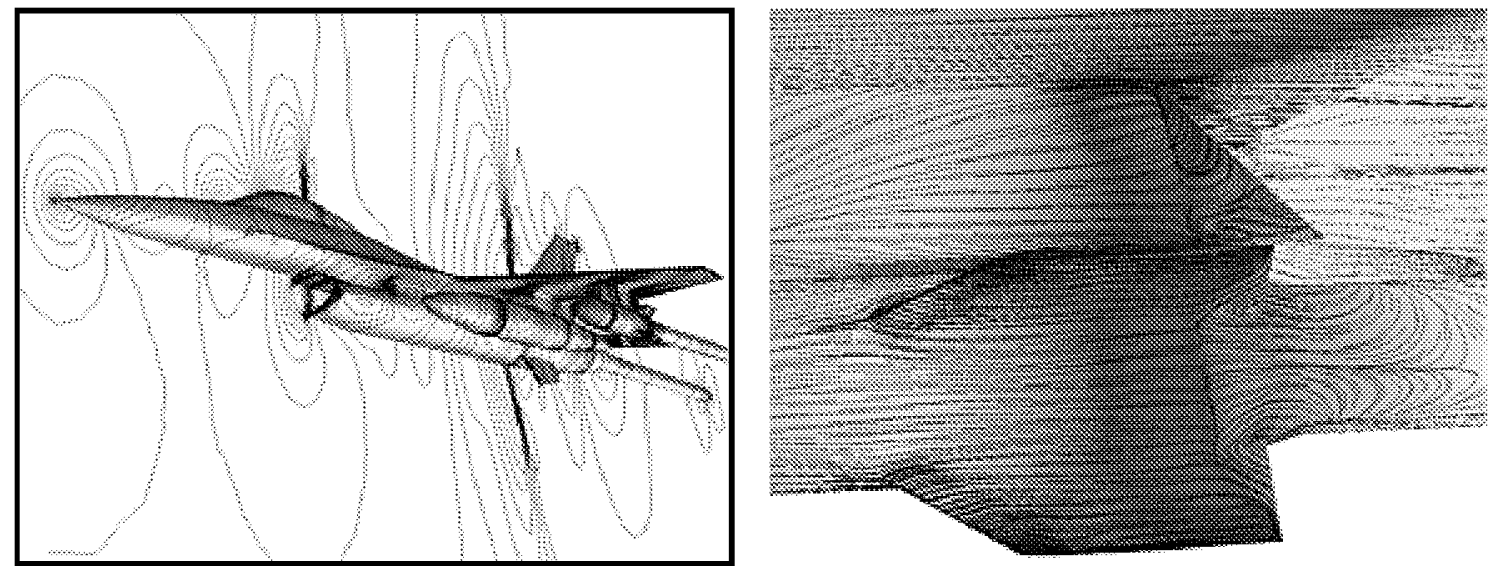

Fig. 7 Navier-Stokes flow solution on complete F-16 aircraft with stores for Mach 0.95, angle of attack 4 degrees, and $\operatorname{Re}_{\text {mac }}=2$ million. (Left - isobars of pressure, Right - surface flow patterns on aft finned-store and pylon.)

store components and in the farfield. The surface flow patterns reveal shock-induced separations on the store afterbody and aft-pylon region.

Force and Moments. Fig. 8 presents a comparison of the experimental force and moments of the store with $\mathrm{N}-\mathrm{S}$ and Euler results from USM3Dns. The plots depict the variation of store normal and side force coefficients (CN and CY, respectively) and the pitch $(\mathrm{Cm})$ and yaw $(\mathrm{Cn})$ moments, in the store body axis system, with angle of attack. Forces are positive upward or inboard from a cockpit perspective. Moments are positive for nose pitching upward or yawing inboard relative to the aircraft. The N-S results are generally in better agreement with the experimental data [11] than the Euler solutions, with the exception of the yawing moment, $\mathrm{Cn}$. The largest benefit to the N-S approach occurs at AOA of 0 degrees. Reference [13] reported difficulties at this AOA with the Euler modeling. Very strong shock interactions were produced by the inviscid equations between the store's aft upper fins and the support pylon which may not be present in real flow. The present N-S solutions produce a more physically correct result by modeling the aft-store-body shock-induced flow separation, as will be shown in the next section.

Surface Pressures. The computed longitudinal distributions of surface pressure coefficient for the finnedstore body are compared with experimental data from Ref. [11] in Fig. 9 for 4 degrees AOA. The top, bottom, inboard, and outboard longitudinal stations correspond to radial locations of 5, 185, 275, and 95 degrees, respectively, measured from the top of the store body and rotating outward away from the fuselage. Values for $x / L_{\text {store }}$ of 0.0 correspond to the store nose, and 1.0 to an aft-body station slightly behind the store fins. The correlation of pressure coefficient is generally good for the bottom, outboard, and inboard longitudinal stations. The poor corre- 

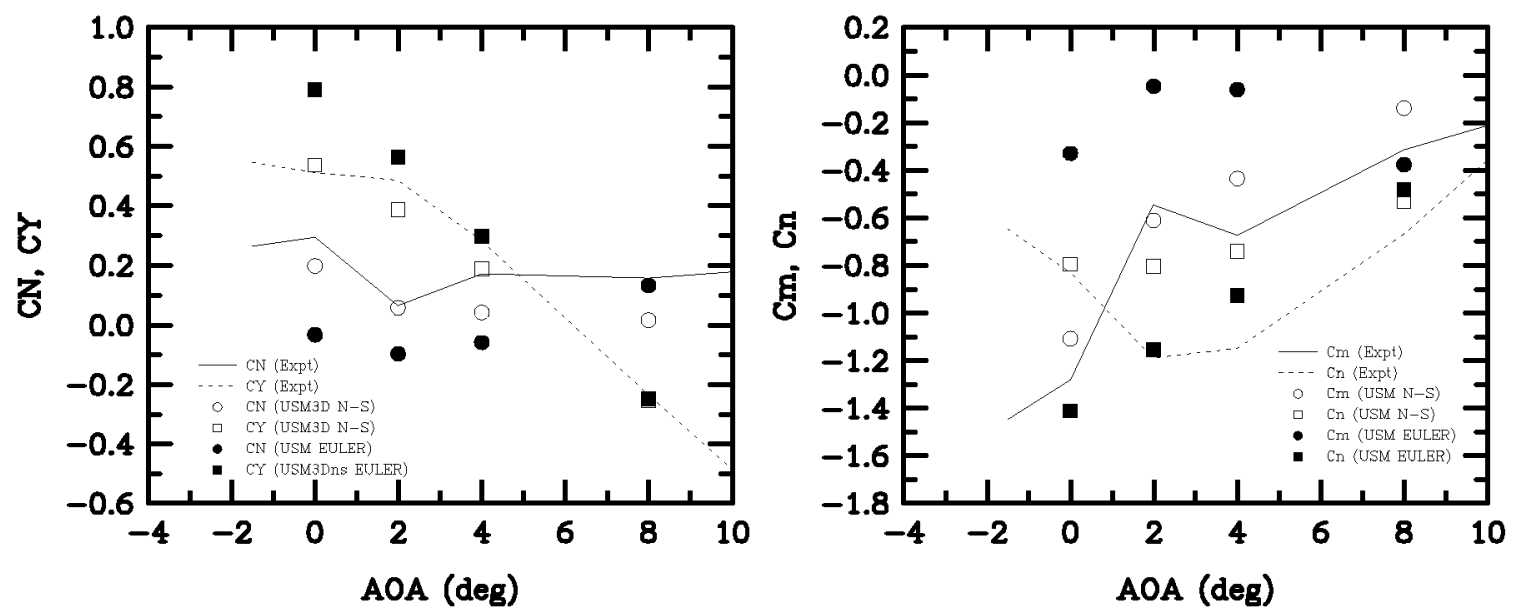

Fig. 8 Variation of store forces and moments with angle of attack, Mach=0.95.
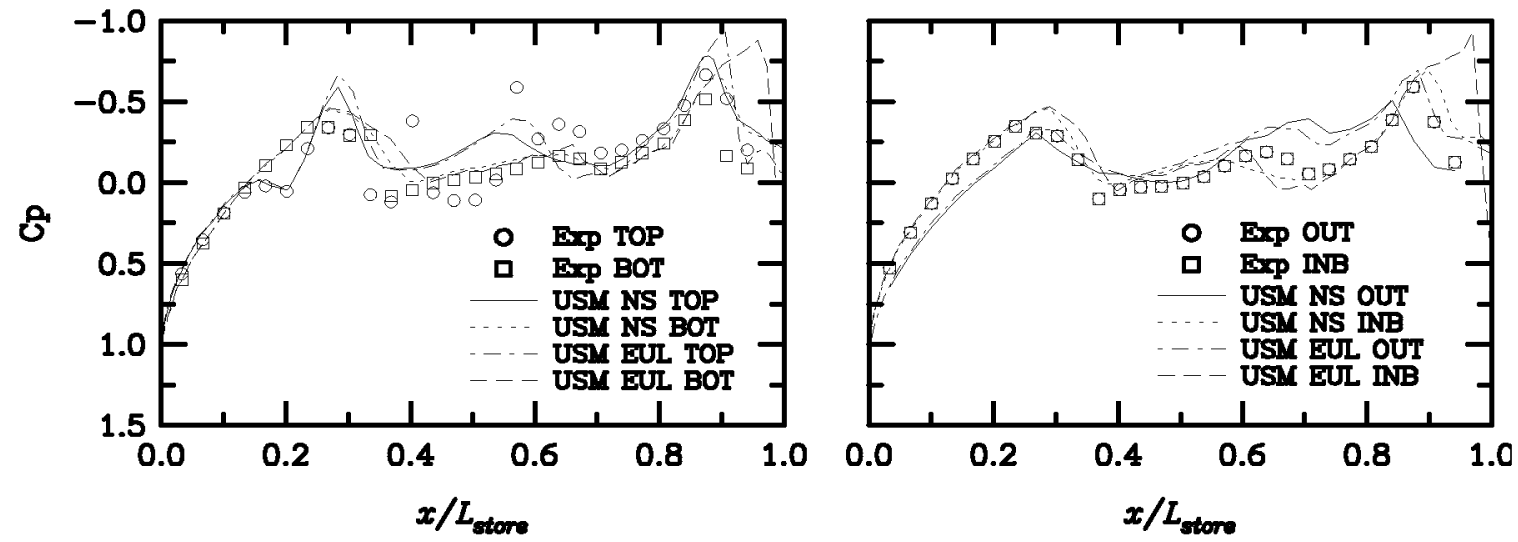

Fig. 9 Longitudinal distribution of surface pressure coefficient on generic finned-store body at Mach 0.95 and angle of attack 4 degrees. (Left - top and bottom, Right - outboard and inboard)

lation along the top station is caused by the interference of sway braces used in the wind tunnel test, but not modeled in the computation, which induce a local suction at store body stations of 43- and 59-percent. Consistent with results from Ref. [13], the Euler solutions predict a rapid expansion followed by a strong shock at the boat-tail of the body. As expected from the N-S solution, the boat-tail separation, evident in the surface flows in Fig. 7, results in a softened expansion in Fig. 9 and brings the aft-shock into better agreement. There is also a generally better agreement of the mid-body compressions for the Navier-Stokes solutions.

A similar, but more dramatic effect can be observed in the fin pressure coefficient distributions in Fig. 10. These longitudinal distributions are plotted along the 50-percent exposed-fin span station for the inboard-upper and outboard-upper fin components. The N-S methodology yields a significant improvement in predicting the shock strength and location on the aft 60-percent of the fin. A further assessment of the effect of grid refinement on the pressure distributions of Figs. 9 and 10 is warranted in future work. However, level of agreement of the present Navier-Stokes comparisons is good considering the relatively small number of tetrahedra $(1,428,779$ cells $)$ used to resolve this complex full aircraft configuration.

\section{CONCLUDING REMARKS}

A review has been presented of the algorithmic features and capabilities of the tetrahedral-based finite-volume Euler and Navier-Stokes flow solver USM3Dns. This code, along with the tetrahedral grid generator, VGRIDns, are primary components of the NASA TetrUSS package which is being used extensively throughout 

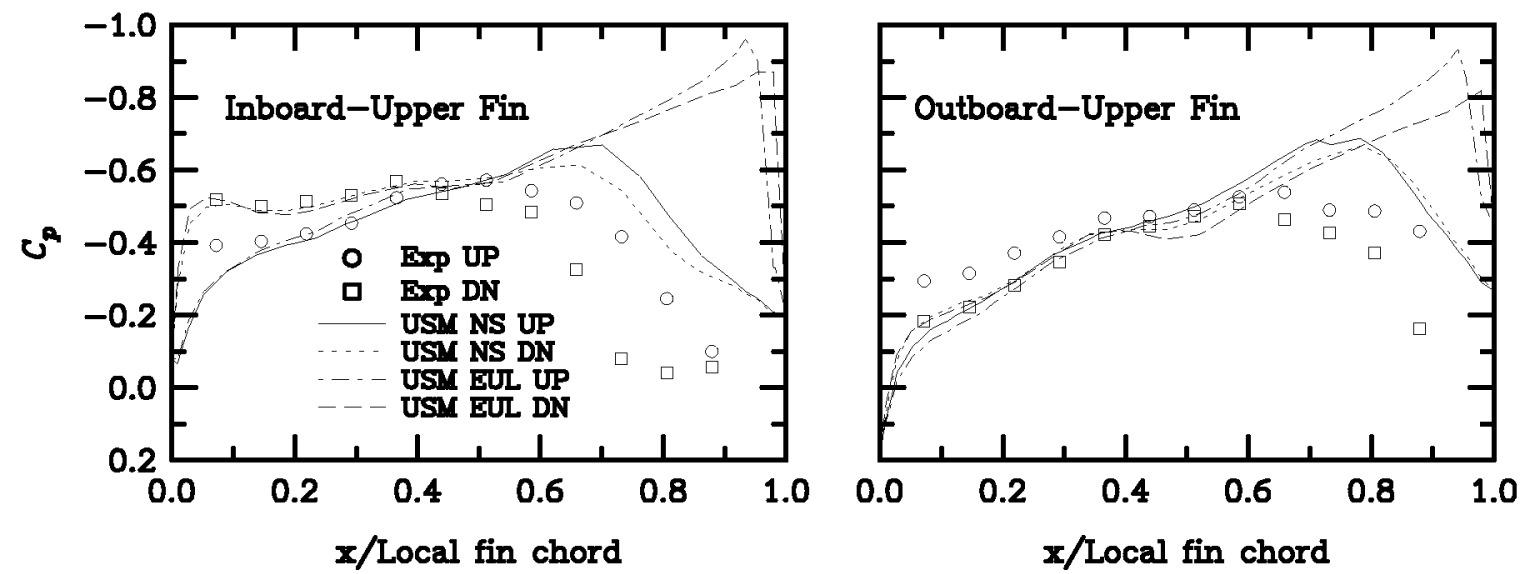

Fig. 10 Longitudinal distribution of surface pressure coefficient on upper inboard and outboard fins on generic store at

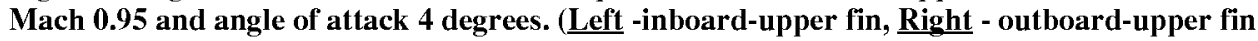

the U.S. for solving complex aerodynamic problems. The Navier-Stokes capability is maturing rapidly as a result of many focused customer applications on a range of configurations.

The present work has addressed the issues of accuracy and robustness of the USM3Dns Navier-Stokes feature using the flat-plate turbulent boundary layer problem, and a full F-16 aircraft with external stores. The code achieves excellent accuracy in predicting the turbulent skin friction coefficient on the flat plate with as few as 15 tetrahedral layers (5 nodes) across the boundary layer using the Spalart-Allmaras one-equation turbulence model with a wall function. The F-16 calculation serves as a good test case for demonstrating robustness and accuracy on a very complex geometry with transonic flow. A reasonably good prediction of force-moments and surface pressures on the generic finned-store body and fins was achieved with as few as 1.4 million tetrahedral cells, and within 2 wallclock hours per solution on a CRAY C90. While many more application studies are needed to increase user confidence in this capability, these results serve to demonstrate the strong potential for tetrahedralbased finite-volume Navier-Stokes methodologies to become a practical computational aerodynamic tool.

\section{ACKNOWLEDGEMENTS}

The authors would like to thank Mr. Steve Kern and Dr. Christopher Bruner of the Navel Air Systems Command for providing the surface definition and experimental data files for the F-16 configuration. The support of Mr. Javier Garriz, ViGYAN, Inc. and Mr. Derek Leitchy, Purdue University, in generating the F-16 viscous grid is also greatly appreciated. The first author would like to extend a special thanks to Dr. David Whitaker, Cray Research, for efficiently multitasking the USM3Dns code. All computations were performed on the Numerical Aerodynamic simulation (NAS) facility at NASA Ames Research Center.

\section{REFERENCES}

1Anderson, W. K.: "Grid Generation and Flow Solution Method for Euler Equations on Unstructured Grids", NASA TM4295, April 1992.

2Anderson, W. K., Rausch, R.D., and Bonhaus, D. L.: "Implicit/Multigrid Algorithms for Incompressible Turbulent Flows on Unstructured Grids", J. Comp. Phys. Vol. 128, 1996, pp. 391-408.

3Baker, T. J.: "Irregular Meshes and the Propagation of Solution Errors," Proceedings 15th International Conference on Numerical Methods in Fluid Dynamics, Monterey, CA, June 1996

4Barth, T. J.: "Numerical Aspects of Computing Viscous High Reynolds Number Flows on Unstructured Meshes", AIAA Paper 91-0721, January 1991.

5Frink, N. T.: "Upwind Scheme for Solving the Euler Equations on Unstructured Tetrahedral Meshes." AIAA Journal, Vol., No. 1, January, 1992, pp. 70-77.

6Frink, N. T.: "Recent Progress Toward a Three-Dimensional Unstructured Navier-Stokes Flow Solver", AIAA 94-0061, January 1994.

7Frink, N. T., Pirzadeh, S., and Parikh, P., “An Unstructured-Grid Software System for Solving Complex Aerodynamic Problems", NASA CP-3291, pp. 289-308, May 9-11, 1995.

8Frink, N. T.:"Tetrahedral Unstructured Navier-Stokes Method for Turbulent Flows", AIAA Journal, Vol. 36, No. 11, pp. 1975-1982, November 1998..

9Hassan, O., Probert, E.J., Morgan K., and Peraire, J., 'Unstructured Tetrahedral Mesh Generation for Three Dimensional 
Viscous Flows", Int. J. Num. Meth. Engng., (University of Wales Swansea Report CR/846/94), 1995 (in press).

10Holmes, D. G., and Connell, S. D., "Solution of the 2D Navier-Stokes Equations on Unstructured Adaptive Grids", presented at the AIAA 9th Computational Fluid Dynamics Conference, June, 1989.

11Hoph, J. C.: "Separation Characteristics of the DWS-24 Dispenser, the MK-84 LDGP, the 370-GAL Tank (E) + Pylon, and the Generic Missile (Metric and Pressure-Instrumented) in the Flow Field of the F-16 Aircraft," AEDC-TSR-94-1, February 1994.

12Jameson, A, Baker, T. J., and Weatherill, N. P.: "Calculation of Inviscid Transonic Flow over a Complete Aircraft", AIAA Paper 86-0103, January 1986.

13Kern, S., and Bruner, C.: "External Carriage Analysis of a Generic Finned-Store on the F-16 Using USM3D", AIAA 96 2456, June 1996

14Löhner, R. and Parikh, P.: "Three-Dimensional Grid Generation by the Advancing Front Method," Int.J.Num.Meth. Fluids 8, 1135-1149, 1988.

15Marcum, D. L., "Generation of Unstructured Grids for Viscous Flow Applications," AIAA-95-0212, 33rd AIAA Aerospace Sciences Meeting, Reno, NV, January 1995.

16Mavriplis, D. J. and Venkatakrishnan, V.: "A 3D Agglomeration Multigrid Solver for the Reynolds-Averaged NavierStokes Equations on Unstructured Meshes”, NASA CR 195073, April 1995.

17Mitchell, C.R.: "Improved Reconstruction Schemes for the Navier-Stokes Equations on Unstructured Meshes", AIAA Paper 94-0642, January 1994.

18Perair, J. Peiro, J., and Morgan, K.: "Finite Element Multigrid Solution of Euler Flows Past Installed Aero-Engines", Computational Mechanics 11, 1993.

19Pirzadeh, S.: "Structured Background Grids for Generation of Unstructured Grids by Advancing-Front Method", $A I A A$ Journal, Vol. 31, No. 2, pp. 257-265, February 1993.

20Pirzadeh, S.: "Unstructured Viscous Grid Generation by Advancing-Layers Method", AIAA Journal, Vol. 32, No. 8, Au gust 1994, pp. 1735-1737.

21Pirzadeh, S.; "Three-Dimensional Unstructured Viscous Grids by the Advancing Front Method", AIAA Journal, Vol. 34, No. 1, January 1996 , pp. $43-49$.

22Pirzadeh, S.: "Progress Toward A User-Oriented Unstructured Viscous Grid Generator", AIAA 96-0031, January 1996.

23Roe, P. L.: Characteristic Based Schemes for the Euler Equations. Annual Review of Fluid Mechanics, Vol. 18, 1986, pp. 337-365.

24Spalart, P.R. and Allmaras, S.R.: "A One-Equation Turbulence Model for Aerodynamic Flows", AIAA Paper 92-0439, January 1992.

25Van Leer, B.: "Flux-Vector Splitting for the Euler Equations", Eighth International Conference on Numerical Methods in Fluid Dynamics, E. Krause, ed., Volume 170 of Lecture Notes in Physics, Springer-Verlag, 1982, pp. 507-512.

26White, F.M.: Viscous Fluid Flow, McGraw-Hill, Inc., ISBN 0-07-069710-8, 1974. 


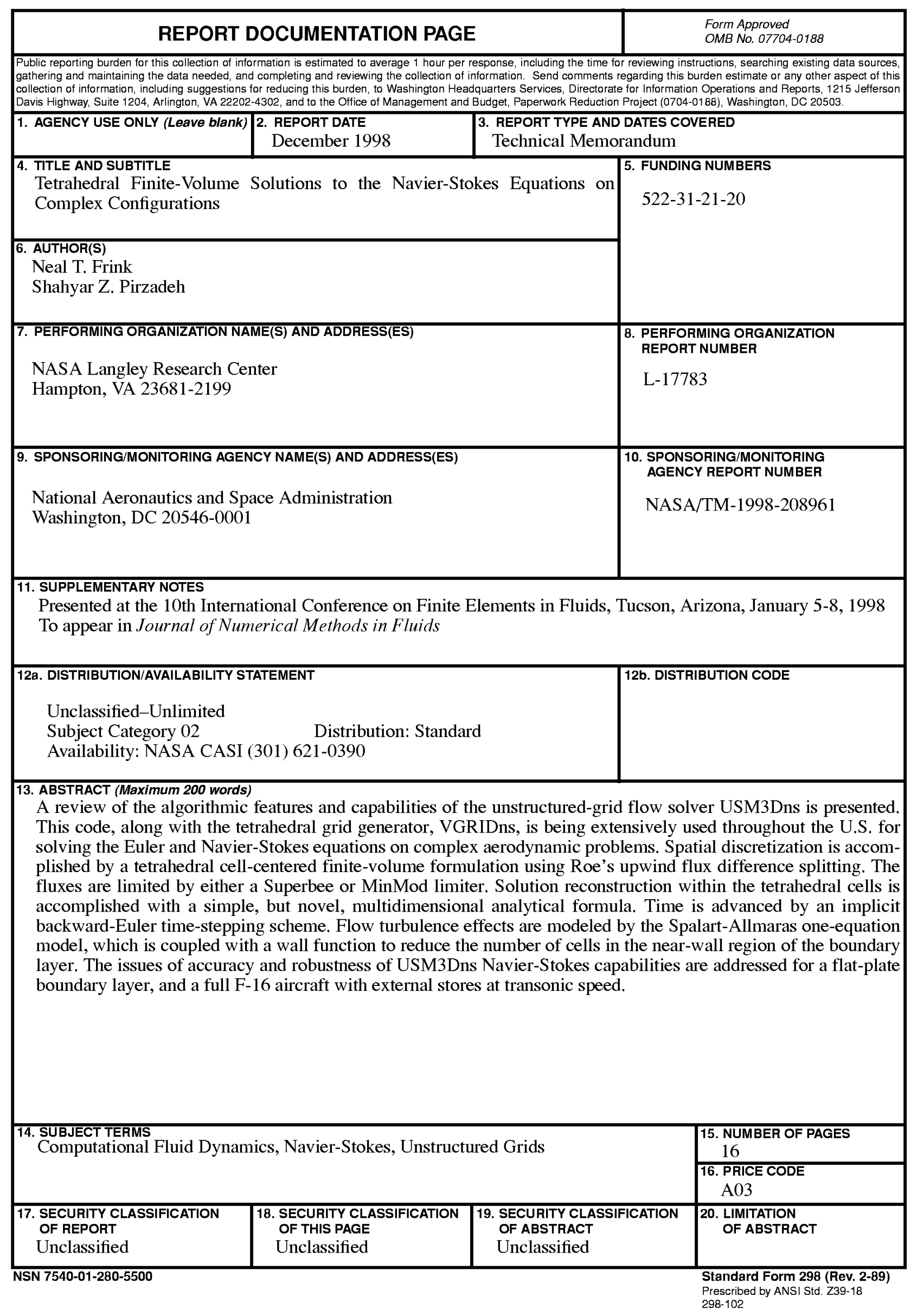

